\title{
Resection of Segments 4, 5 and 8 for a Cystic Liver Tumor Using the Double Liver Hanging Maneuver
}

\author{
Atsushi Nanashima Yorihisa Sumida Takafumi Abo \\ Takashi Nonaka Hideyori Sengyoku Terumitsu Sawai \\ Toru Yasutake Takeshi Nagayasu \\ Division of Surgical Oncology, Department of Translational Medical Sciences, \\ Nagasaki University Graduate School of Biomedical Sciences, Nagasaki, Japan
}

\section{Key Words}

Central liver $\cdot$ Hepatic resection $\cdot$ Double liver hanging maneuver

\begin{abstract}
To achieve complete anatomic central hepatectomy for a large tumor compressing surrounding vessels, transection by an anterior approach is preferred but a skillful technique is necessary. We propose the modified technique of Belghiti's liver hanging maneuver (LHM). The case was a 77-year-old female with a 6-cm liver cystic tumor in the central liver compressing hilar vessels and the right hepatic vein. At the hepatic hilum, the spaces between Glisson's pedicle and hepatic parenchyma were dissected, which were (1) the space between the right anterior and posterior Glisson pedicles and (2) the space adjacent to the umbilical Glisson pedicle. Two tubes were repositioned in each space and 'double LHM' was possible at the two resected planes of segments 4, 5 and 8 . Cut planes were easily and adequately obtained and the compressed vessels were secured. Double LHM is a useful surgical technique for hepatectomy for a large tumor located in the central liver.
\end{abstract}

\section{Introduction}

Large liver tumors that expand in the central liver of segments 1, 4, 5 and 8 often compress the intra- or extrahepatic vessels and adjacent organs [1]. In such tumors, central liver resection such as right paramedian sectoriectomy (segments 5 and 8) or resection of segments 4,5 and 8 is carried out and the anterior approach would be selected [2]. To achieve anatomic resection, it is important to ensure adequate exposure of 
the transection plane or the hepatic vein [3]. Furthermore, in case of a large liver tumor compressing the right hepatic vein or the umbilical portal pedicle, it may be difficult to adequately expose the transection planes. The liver hanging maneuver (LHM) proposed by Belghiti et al. [4] is a useful technique for the anterior approach to reduce the time required for hepatic transection and blood loss in the right or left hemi-hepatectomy [5]. Furthermore, an adequate transection plane might be required using the LHM. The application of this technique for anatomical resections has been reported recently [6] and we also reported the 'double LHM' by hanging two transection planes for the right paramedian sectoriectomy [7]. We believe that the double LHM is useful for resection of the central liver in large liver tumors and here we report this technique for resection of segments 4, 5 and 8 in patients with a large benign tumor.

\section{Surgical Techniques}

The patient undergoes a J-shaped incision laparotomy (upper median plus right-sided transverse incision to the 10th intercostal space) in the supine position [8]. The falciform ligament is cut and we subsequently expose the bifurcation of the right, middle, and left hepatic veins, and the anterior surface of the vena cava. Mobilization of the liver is not carried out. LHM has been basically followed by Belghiti's method [4]. We describe this technique in brief. The space between the right and middle hepatic vein is dissected using a right angled clamp first. From the same space, the space with loose connective tissue between the anterior surface of the vena cava and the paracaval caudate lobe is dissected for $3 \mathrm{~cm}$ using a long right angled clamp for the renal artery. Subsequently, the space between the vena cava and the infra-hepatic caudate process is dissected and a few short hepatic veins are divided. The loose tissue is dissected for $3 \mathrm{~cm}$ using a long, light and curved Kelly clamp. A 10-Fr nasogastric tube (tube A) is inserted between the right and middle hepatic vein, and then easily passed through the dissected space [7] (fig. 1). Another 10-Fr tube (tube B) is inserted between the right and left hepatic veins, and its tip is placed in the gap between segments 2 and 3 and the caudate lobe (fig. 1).

At the hepatic hilum, the spaces between Glisson's pedicle and hepatic parenchyma are dissected using a right angled clamp, which are (1) the space between the right anterior and right posterior Glisson pedicles and (2) the space adjacent to the umbilical Glisson pedicle [7]. The right edge of the caudate lobe is cut and tube $\mathrm{A}$ is placed in the space between the right anterior and right posterior Glisson pedicles by the tape-repositioning technique reported by Kokudo et al. [9] (fig. 2). Tube B is positioned in the space adjacent to the umbilical Glisson pedicle (fig. 2). Then the double LHM is prepared using the nasogastric tubes. The hepatic transection is performed using a combination of the crush clamping method and use of the ultrasonic dissector during the intermittent occlusion of hepatic inflow (15 min occlusion and 5 min de-clamping) using Pringle's maneuver [10]. The tubes are always pulling up during the transection and the direction of transection is always targeted toward the hanged tube. Transection to the anterior aspect of the vena cava can easily be performed under the hanging tube.

\section{Patient}

A 77-year-old female with abdominal discomfort was found to have a large and cystic liver tumor measuring $6 \mathrm{~cm}$ in diameter occupying segments $1,4,5$ and 8 on computed tomography scan (fig. 3 ). By the image diagnosis, ruling out malignant liver tumors such as a cystadenocarcinoma was difficult. The tumor compressed the right hepatic vein and Glisson's pedicle at the hepatic hilum, and a resection of segments 4, 5 and 8 including a part of the caudate lobe was planned. We applied the double LHM in 
the present case. The Glisson pedicles in the right paramedian sector and in segment 4 were divided. As shown in figure $4 \mathrm{a}$ and $\mathrm{b}$, an adequate transection plane could be obtained along the umbilical pedicle and along the right hepatic vein using this technique because the remnant liver (segments $6+7$ and segments $2+3$ ) was rotated to the counter side from the resected liver, including the tumor, upon lifting the nasogastric tube during transection. The hanging tube was always pulled up during transection and the direction of transection was always targeted toward the tube on the vena cava. The adhesive spaces between tumor and hilar bile ducts were carefully dissected. Finally, the caudate lobe was dissected by the replacement of the nasogastric tube ( $\underline{\text { fig. }}$. $)$ and the middle hepatic vein was cut and divided. The resection of segments 4,5 and 8 was completed (fig. 6). The total transection time was $52 \mathrm{~min}$, the operating time was $11 \mathrm{~h} 31 \mathrm{~min}$, and blood loss was 1,020 $\mathrm{ml}$ but required no red blood cell transfusion.

\section{Discussion}

Central liver resection such as bisegmentectomy (segments 4,5 and 8 ), resection of segment 4 , and right paramedian sectoriectomy (segments 5 and 8 ) are often selected as anatomic resection for liver tumors [3]. Two wide areas for parenchymal transection are necessary in such cases and these types of hepatectomies are skillful operative procedures in liver surgery. Such hepatectomy often requires a longer time for transection or is associated with a larger amount of bleeding, relative to other anatomic resections, and may be associated with functional liver damage or postoperative complications [11]. Thus, it is necessary to reduce transection time or intraoperative bleeding by innovative techniques. In central liver resections, the key markers of transected lines for transection are hepatic veins and Glisson's pedicles. Usually, transected planes are confirmed by intraoperative ultrasonography during parenchymal transection [12].

LHM has been applied not only for right hemihepatectomy but also left hepatectomy [13] and isolated caudate lobectomy [14]. Thus, LHM can be applied for various hepatic resections. However, to our knowledge, application of LHM has not been fully reported yet for central hepatectomy [6]. Our modified technique of the double LHM was previously reported in a case with hepatocellular carcinoma in the right paramedian sector [7]. In such a malignant liver tumor, mobilization of the remnant liver might be avoided because liver rotation has the disadvantage of circumventing tumor dissemination [15]. The right paramedian sectoriectomy was easily performed using this modified technique [7]. In the present case, the tumor compressed the surrounding vessels and the bottom of the tumor was located in a part of the paracaval portion of the caudate lobe. Dissection of these spaces in the deeper part of the liver was expected to be difficult by the conventional procedure. We deduce that LHM is adequate to resolve problems associated with central hepatectomy. Since the transected area is compressed by LHM, the transection time or parenchymal bleeding might be reduced compared to the non-hanging procedure. Another advantage of LHM is confirmation of the appropriate cut line during transection [7]. By placing a hanging tape or tube, the transected line can always be targeted to this tape. As the tape was placed adjacent to the hepatic vein, appropriate transection could be accomplished by LHM. In resection of segments 4, 5 and 8 , transection lines were the line along the right hepatic vein and the line on the adjacent right side of the umbilical Glisson pedicle. The inserted tubes were replaced by passing between Glisson's pedicle and liver parenchyma by applying the gradual taperepositioning technique proposed by Kokudo et al. [9]. By this 'double LHM' technique, the transected planes are always stable and the surgeons can target the position of the tubes during transection. Then the estimated anatomic liver resection is performed easily. Our experience indicates that the LHM and its application such as double LHM is a useful operative technique in anatomic hepatic resections for trainees in terms of education in liver surgery. 


\section{Conclusion}

A large hepatoma could also compress the adequate transection plane or the main vessels in the preserved side of the liver. Taking advantage of lifting the resected liver or the weight of the tumor, the preserved side of the liver was gradually rotated by the LHM and an adequate transection plane could be opened, regardless of tumor compression. Since the tube in the space between the liver and vena cava is always fixed, the surgeon can easily transect toward the tube. In summary, our present experience indicates that LHM can be applied for central hepatectomy in segments 4, 5 and 8 and could be considered a key operative technique in liver resection for large liver tumors.

Fig. 1. Schematic diagram illustrating the intial insertion and placement of the nasogastric (NG) tubes (tubes A and B).

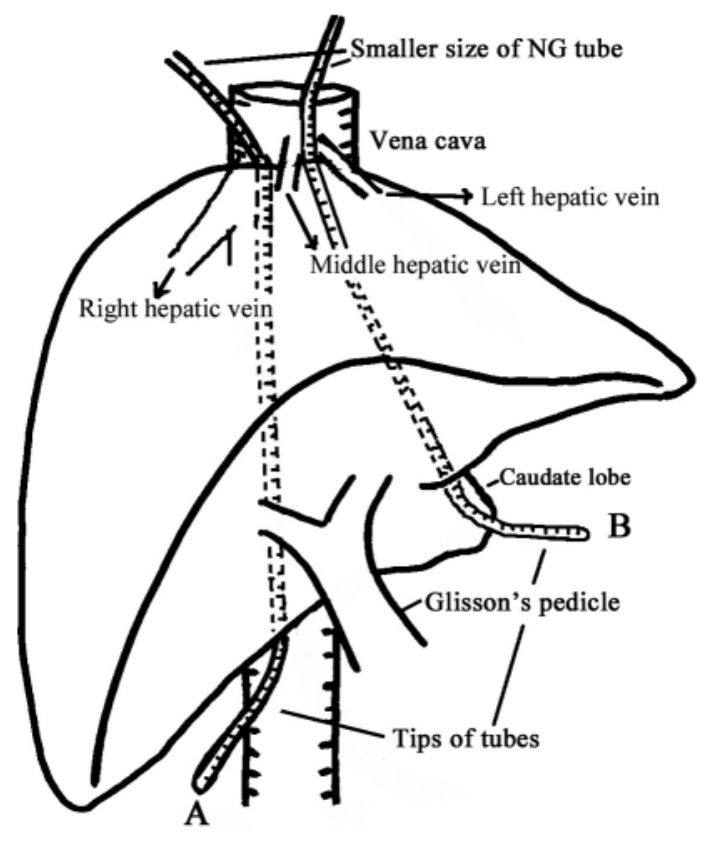


Fig. 2. Schematic diagram illustrating repositioning of tubes $A$ and $B$ in the space between the right anterior and posterior Glisson pedicle, and adjacent to the umbilical Glisson pedicle. Thin arrows: direction of the tube passage; thick arrow: cut of the caudate lobe.

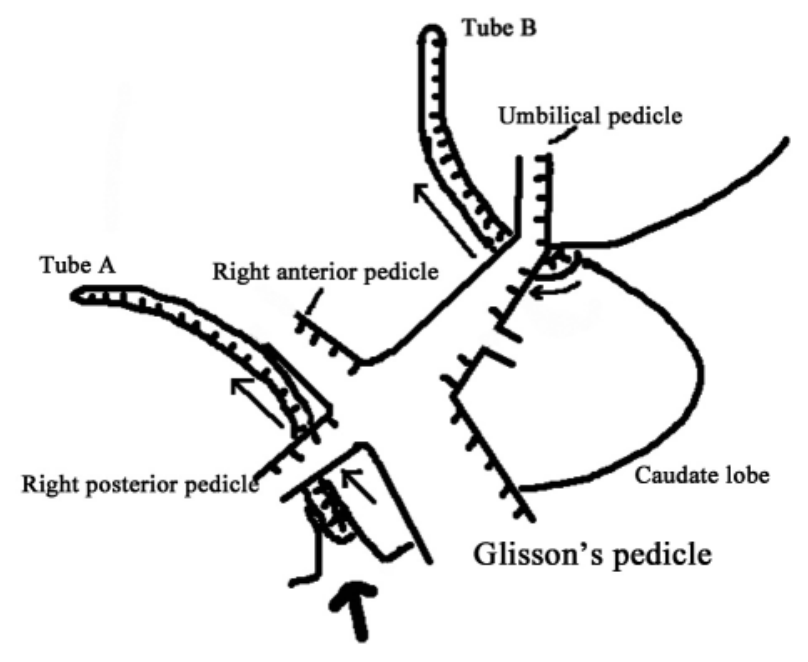

Fig. 3. Computed tomography finding of a large cystic tumor occupying the central liver and compressing the right hepatic vein (arrow) and the surrounding Glisson pedicle (arrowheads).

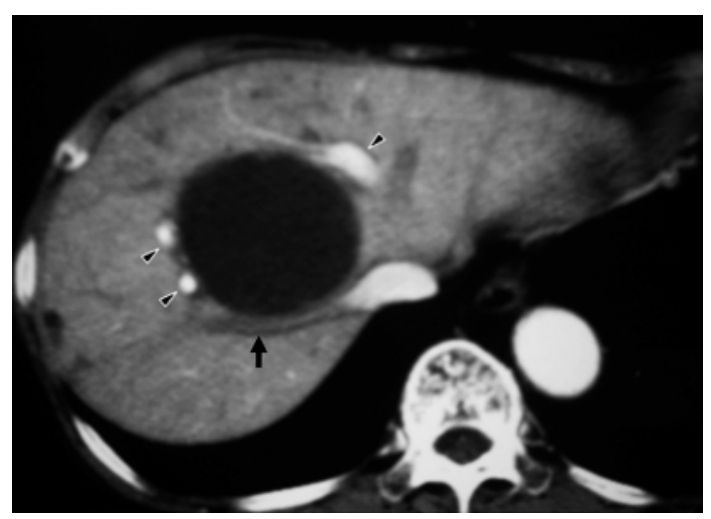




\begin{tabular}{c|l|l|l}
$\begin{array}{c}\text { Case Reports in } \\
\text { Gastroenterology }\end{array}$ & $\begin{array}{l}\text { Case Rep Gastroenterol 2008;2:60-66 } \\
\text { D01: 10.1159/000119029 }\end{array}$ & Published online: March 11, 2008 & $\begin{array}{l}\text { O 2008 S. Karger AG, Basel } \\
\text { ISSN 1662-0631 } \\
\text { www.karger.com/crg }\end{array}$ \\
\hline
\end{tabular}

Fig. 4. The resected liver, including the tumor, upon lifting the nasogastric tube during transection. Two adequate transection planes could be obtained along the umbilical pedicle and along the right hepatic vein using this technique. a Cut plane between segment 4 and segments $2+3$. b Cut plane along the right hepatic vein (arrow).
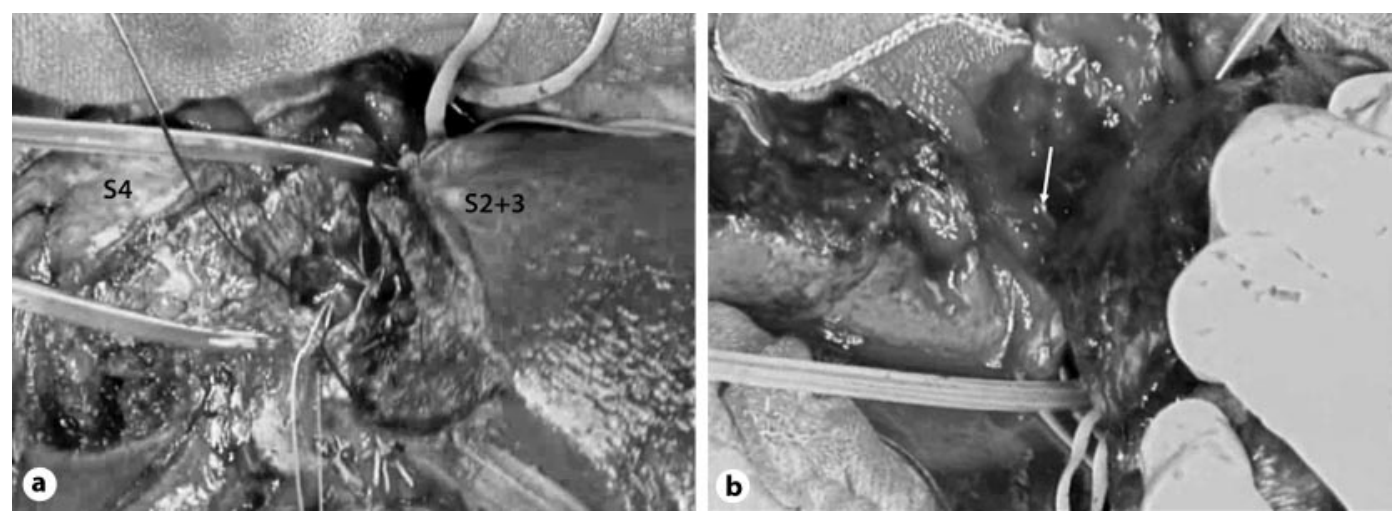

Fig. 5. The caudate lobe was dissected by replacement of the nasogastric tube at the bottom of tumor.

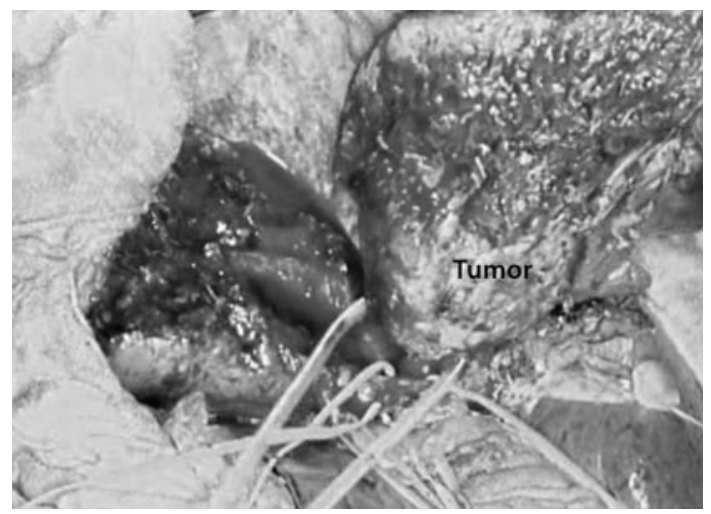

Fig. 6. The view of the transected edge after resection of segments 4, 5 and 8 . The right hepatic vein (arrow) and hilar Glisson's pedicle (arrowhead) were clearly exposed.

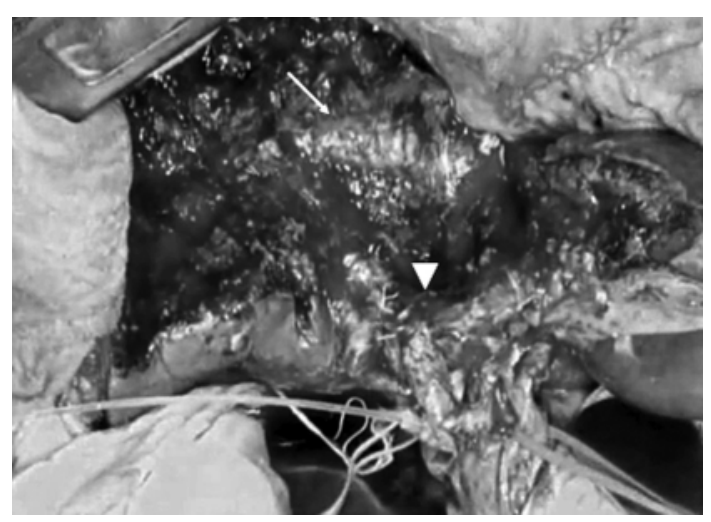




\section{References}

1 Tanaka K, Nishimura A, Takenaka K, Yamada K, Ishibe R, Ogata S, et al: Extended central bisegmentectomy - an en bloc resection of hepatic segments 4, 5, 8 and 7: report of a case. Surg Today 1994;24:170-172.

-2 Hu RH, Lee PH, Chang YC, Ho MC, Yu SC: Treatment of centrally located hepatocellular carcinoma with central hepatectomy. Surgery 2003;133:251-256.

-3 Hasegawa K, Kokudo N, Imamura H, Matsuyama Y, Aoki T, Minagawa M, et al: Prognostic impact of anatomic resection for hepatocellular carcinoma. Ann Surg 2005;242:252-259.

-4 Belghiti J, Guevara OA, Noun R, Saldinger PF, Kianmanesh R: Liver hanging maneuver: a safe approach to right hepatectomy without liver mobilization. J Am Coll Surg 2001;193:109-111.

5 Gaujoux S, Douard R, Ettorre GM, Delmas V, Chevallier JM, Cugnenc PH: Liver hanging maneuver: an anatomic and clinical review. Am J Surg 2007;193:488492.

-6 Kim SH, Park SJ, Lee S, Lee WJ, Park JW, Hong EK, et al: Various liver resections using hanging maneuver by three Glisson's pedicles and three hepatic veins. Ann Surg 2007;245:201-205.

7 Nanashima A, Sumida Y, Abo T, Yasutake T, Nagayasu T: Right paramedian sectoriectomy using the double liver hanging maneuver. Surgery 2007;142:430431.

8 Sato H, Sugawara Y, Yamasaki S, Shimada K, Takayama T, Makuuchi M, et al: Thoracoabdominal approaches versus inverted $\mathrm{T}$ incision for posterior segmentectomy in hepatocellular carcinoma. Hepatogastroenterology 2000;47:504-506.

-9 Kokudo N, Sugawara Y, Imamura H, Sano K, Makuuchi M: Sling suspension of the liver in donor operation: a gradual tape-repositioning technique. Transplantation 2003;76:803-807.

10 Man K, Fan ST, Ng IO, Lo CM, Liu CL, Wong J: Prospective evaluation of Pringle maneuver in hepatectomy for liver tumors by a randomized study. Ann Surg 1997;226:704-711.

-11 Nanashima A, Sumida Y, Abo T, Tanaka K, Takeshita H, Hidaka S, et al: Clinicopathological and intraoperative parameters associated with postoperative hepatic complications. Hepatogastroenterology 2007;54:839-843.

-12 Torzilli G, Montorsi M, Del Fabbro D, Palmisano A, Donadon M, Makuuchi M: Ultrasonographically guided surgical approach to liver tumours involving the hepatic veins close to the caval confluence. Br J Surg 2006;93:1238-1246.

13 Suh KS, Lee HJ, Kim SH, Kim SB, Lee KU: Hanging maneuver in left hepatectomy. Hepatogastroenterology 2004;51:1464-1466.

14 Kim SH, Park SJ, Lee S, Lee WJ, Park JW, Kim CM: Isolated caudate lobectomy using the hanging maneuver. Surgery 2006;139:847-850.

15 Yamanaka N, Okamoto E, Fujihara S, Kato T, Fujimoto J, Oriyama T, et al: Do the tumor cells of hepatocellular carcinomas dislodge into the portal venous stream during hepatic resection? Cancer 1992;70:2263-2267. 